\title{
Anabases
}

ANABASES Traditions et réceptions de l'Antiquité

$8 \mid 2008$

Varia

\section{Luigi-Alberto SANCHI, Les Commentaires de la langue grecque de Guillaume Budé. L'œuvre, ses sources, sa préparation}

\section{Françoise Frazier}

\section{(2) OpenEdition}

\section{Journals}

Édition électronique

URL : http://journals.openedition.org/anabases/240

DOI : 10.4000/anabases. 240

ISSN : 2256-9421

\section{Éditeur}

E.R.A.S.M.E.

\section{Édition imprimée}

Date de publication : 1 octobre 2008

Pagination : 277-279

ISSN : 1774-4296

\section{Référence électronique}

Françoise Frazier, «Luigi-Alberto SANCH, Les Commentaires de la langue grecque de Guillaume Budé L'œuvre, ses sources, sa préparation », Anabases [En ligne], 8 | 2008, mis en ligne le 01 juillet 2011. consulté le 22 septembre 2020. URL : http://journals.openedition.org/anabases/240 ; DOI : https:// doi.org/10.4000/anabases. 240

Ce document a été généré automatiquement le 22 septembre 2020

(c) Anabases 


\title{
Luigi-Alberto SANCHI, Les Commentaires de la langue grecque de Guillaume Budé. L'œuvre, ses sources, sa préparation
}

\author{
Françoise Frazier
}

\section{RÉFÉRENCE}

Luigi-Alberto SANCHI, Les Commentaires de la langue grecque de Guillaume Budé. L'œuvre, ses sources, sa préparation, préface de Brigitte Mondrain, Travaux d'Humanisme et Renaissance 410, Genève, Droz, 2006, VIII-328 p.

127,51 euros / ISBN 2-600-01040-8.

1 Avec cette étude, tirée d'une thèse de doctorat, est mis à la disposition des seiziémistes, mais aussi des hellénistes et des historiens, un très précieux instrument de travail sur les Commentaires de la langue grecque de G. Budé, paru en 1529 chez Josse Bade, ouvrage historiquement important, mais dont le caractère très "touffu », déjà déploré par Érasme, avait jusqu'à présent quelque peu rebuté les savants. L-AS. comble donc un vide et il donne avec beaucoup de clarté et de précision tous les éléments importants pour juger de l'entreprise de Budé.

2 La composition même de son étude en témoigne, qui se partage en deux parties à peu près égales, une étude en sept chapitres de la situation historique, de la genèse et de la préparation de l'œuvre (p.1-153) suivie d'une copieuse bibliographie (p.155-170) et une série de dix annexes, qui donnent une idée précise de l'œuvre elle-même (p.174-307) : on signalera en particulier, après la description détaillée du contenu des Commentaires (Annexe I), l'Annexe II, très précieux répertoire des auteurs grecs cités, qui donne le nom de l'auteur en latin, le genre littéraire, l'époque, la référence de la première occurrence dans les Commentaires et le nombre de citations, puis la disponibilité connue 
parmi les livres de Budé et la présence dans les Carnets autographes, enfin la liste des titres cités, dans la forme et l'ordre des Commentaires. Ce répertoire est complété par un index des auteurs (Annexe III) et un regroupement des auteurs par genre (Annexe IV), " outil de travail magnifique ", selon les termes de B. Mondrain, qui a préfacé l'ouvrage (p.VII). S'ajoutent encore, pour faciliter l'appréhension de l'œuvre, un index des digressions (Annexe VIII), une liste des corrections des textes dans les Commentaires (Annexe IX) et une table des correspondances des éditions de 1529 et 1557 (Annexe 10). L'ensemble est enfin enrichi de neuf illustrations qui font « voir » au lecteur les carnets et l'exemplaire de Budé de 1529 annoté de sa main (p. 311-319).

3 Les deux premiers chapitres (1. «Les études grecques en France à l'époque de Budé » et 2. "Les Commentaires et les chaires royales: la préface à François $\mathrm{I}^{\mathrm{er}}$ ), montrent comment les données biographiques se superposent avec les débuts de l'hellénisme en France, inscrits dans le cadre de l'émulation avec le Quattrocento italien. La préface de même, éditée pour la première fois, privilégie sur la simple présentation une apologie des études grecques, qui est mise en rapport avec « une situation concrète »: L.-A. S. souligne ainsi «l'importance historique d'un texte appelé à relancer, de façon décisive, à la cour aussi bien que dans les milieux humanistes français, le débat sur la création d'une institution de prestige et de haut niveau dans la capitale du royaume » (p. 37).

Les trois chapitres suivants s'attachent à la constitution de l'œuvre: le chapitre 3 en considère "la genèse ", le chapitre 4 décrit "la disposition des matériaux lexicographiques ", tandis que le chapitre 5 , long de plus de cinquante pages, s'attache aux "digressions", qui "s'ajoutent aux autres discontinuités du texte». Budé, conscient des problèmes de composition ainsi posés, a préféré le recours à des renvois internes au regroupement des éléments linguistiques de même ordre. Après une vue d'ensemble, L-A.S. traite des grands domaines ainsi développés par Budé, grammaire (p. 67-81), rhétorique et stylistique (p. 76-81), philosophie (p. 81-87), théologie chrétienne (p. 87-92), droit (p. 92-94), realia (p. 94-96), soulignant en conclusion l'utilité d'une lecture continue des Commentaires, et la volonté de l'auteur de donner plus qu'un simple dictionnaire bilingue. Selon une image heureuse de L-A S., «il oriente la navigation du lecteur sur l'océan lexical ; il intervient à point nommé pour montrer les profondeurs cachées derrière celle-ci; il propose des plongées dans des régions importantes du savoir sous-jacent au vocabulaire» (p. 97). L.-A. S. analyse ensuite en détail (p. 97-111) la « digression cicéronienne » (col. 1419, 11-1451, 54), qui constitue le cœur de la troisième partie des Commentaires : passage plein de brio, où Budé s'oppose, comme Érasme, au cicéronianisme italien, mais en adoptant une perspective générale et linguistique qui se distingue des intérêts humains, spirituels et esthétiques du Ciceronianus, il constitue en quelque sorte le faite argumentatif de l'œuvre, parallèle étoffé de la brève polémique initiale contre Valla, qui se reflète encore dans les deux pièces liminaire et finale, écrites en grec - elles sont données dans les annexes $\mathrm{V}$ pour la préface, traduite dans le texte, et VI pour la postface, traduite dans l'Annexe VII. Préoccupé du déclin littéraire auquel la rigidité des cicéroniens lui semble condamner le latin, Budé montre tout l'enrichissement que l'on peut aussi tirer du grec sans négliger pour autant la langue vernaculaire, dernier point analysé dans cet important chapitre (p.111-119). À travers les étymologies franco-grecques c'est la question linguistique nationale qui est posée, prélude à la promulgation de l'édit de VillersCotterêts (1539), auquel il participera, et préfiguration de la manière même dont la 
modernisation du français sera conçue par les cercles intellectuels dans la seconde moitié du siècle.

Les deux derniers chapitres s'efforcent enfin de préciser les conditions de travail de l'humaniste. Le chapitre 6 s'interroge sur « les lectures grecques de Budé » et propose les «Premiers éléments d'une reconstitution de sa bibliothèque d'après l'œuvre des Commentaires ». L.-A.S. confronte les données internes et ce que l'on sait de l'état des éditions grecques imprimées ou des manuscrits dont a pu disposer Budé, tout en marquant les limites de ce premier aperçu, nécessairement partiel, ne serait-ce qu'en raison des choix lexicologiques opérés par Budé. Enfin le chapitre 7 s'attache à "l'atelier de l'humaniste. Publications et carnets ». Les carnets autographes, conservés à Genève, présentent des écarts avec la rédaction finale des Commentaires, qui montrent le large travail de dépouillement à la base de la réflexion. Attentif en particulier à l' Etymologicum Magnum, Budé s'y intéresse à des mots rares, propose des émendations, annote abondamment livres et carnets. Là encore, une synthèse des quelque 1800 folios conservés est d'autant plus délicate et prématurée que chaque carnet paraît indépendant. Il semble en tout cas qu'il faille renoncer à les utiliser pour préciser le parcours chronologique des études de Budé. Ils permettent néanmoins de préciser la pratique de Budé par rapport à la tradition humaniste. Sa recherche est centrée sur l'érudition et la critique de textes a d'abord des visées historiques. La prose y domine sur la poésie, avec une répartition équilibrée entre philosophie, histoire-géographie, éloquence et théologie. On voit là "une réalisation assez fidèle de l'idéal de l' encyclopédie de Budé » (p. 147), un idéal totalisant nettement différent de la tradition médiévale du trivium et du quadrivium, mais aussi de l'humanisme italien, plus intéressé par la poésie. L'accent est ici mis davantage sur les textes religieux et théologiques - en quoi Budé s'accorde avec l'humanisme septentrional. La tradition byzantine a elle aussi sa place, qui découle tout naturellement du contact établi par Budé avec ses premiers maîtres, Georges Hermonyme et Jean Lascaris. Enfin il faut aussi souligner la genèse juridique des Commentaires: commencée par les textes latins, l'étude des textes juridiques s'est poursuivie dans le domaine grec, s'élargissant, par le biais de la lexicologie, à l'ensemble de la culture hellénisante de l'Antiquité.

6 "Quel fut le sort des Commentaires? ? : ainsi s'ouvre une conclusion (p. 149-153) qui, audelà des critiques de forme d'Érasme, esquisse les premiers linéaments d'une histoire de la réception des Commentaires, rappelant le rayonnement de l'œuvre, la science reconnue de son auteur, les trois éditions du Commentaires qui parurent en 1530 à Bâle, Venise et Cologne, son usage au Collège et la dette revendiquée par plus d'un humaniste, au premier rang desquels figure Henri Estienne, dont le Thesaurus de 1572 éclipsa sans doute les Commentaires, mais en est aussi, d'une certaine manière, l'héritier. Au-delà de cette œuvre, L.-A. S. souligne enfin la liberté que Budé a pu trouver dans son statut de fonctionnaire de haut rang, et non de professeur, pour repenser radicalement les méthodes d'enseignement et de recherche, la nécessité d'éviter les divisions entre disciplines et langues littéraires quand on étudie les humanistes de la Renaissance, enfin la question posée à notre époque même de la relation à maintenir entre les idées et les faits. Cette large perspective finale dit assez tout l'intérêt d'une étude qui associe examen scrupuleux du travail de l'humaniste, éclairé par l'analyse comme par des répertoires précieux, et réflexion sur le sens même de l'humanisme, pour la Renaissance comme pour nous. 


\section{AUTEURS}

FRANÇOISE FRAZIER

Université de Paris Ouest-Nanterre

frazierfrancoise@yahoo.fr 